E.A. Kotikova, M.N. Rybakov

\title{
Kripke Incompleteness of First-order Calculi with Temporal Modalities of CTL and Near Logics ${ }^{1}$
}

\author{
Kotikova Ekaterina Alexandrovna \\ Faculty of Mathematics, Tver State University. \\ Zhelabova 33, Tver, 170100, Russian Federation. \\ e-mail: kate-sunflower91@mail.ru \\ Rybakov Mikhail Nikolayevich \\ Faculty of Mathematics, Tver State University. \\ Zhelabova 33, Tver, 170100, Russian Federation. \\ e-mail: m_rybakov@mail.ru
}

\begin{abstract}
We study an expressive power of temporal operators used in such logics of branching time as computational tree logic or alternating-time temporal logic. To do this we investigate calculi in the first-order language enriched with the temporal operators used in such logics. We show that the resulting languages are so powerful that many 'natural' calculi in the languages are not Kripke complete; for example, if a calculus in such language is correct with respect to the class of all serial linear Kripke frames (even just with constant domains) then it is not Kripke complete. Some near questions are discussed.
\end{abstract}

Keywords: Kripke incompleteness, first-order logic, computational tree logic, alternatingtime temporal logic, recursive enumerability

\section{Preliminaries}

The subject under our consideration is an expressive power of temporal modalities used in such logics as CTL $^{*}$, CTL, LTL, ATL*, ATL, etc., see $[1,7,9,18]$. Here we dwell on the modalities of CTL but the argumentation below remains to be applicable for other logics, too (and we shall show this).

All the logics mentioned above are defined via Kripke semantics, and are Kripke complete by their definitions. It is known that they are decidable and even that the corresponding decision problems are complete in such classes as PSPACE (for LTL, see [21]), EXPTIME (for CTL and ATL, see $[11,24]$ ), and 2-EXPTIME (for $\mathbf{C} \mathbf{T L}^{*}$ and $\mathbf{A T L}^{*}$, see $[12,19,23]$ ). As a corollary of their decidability, they have decidable axiomatizations.

${ }^{1}$ The work is supported by RFBR, projects $13-06-00861$ and $14-06-00298$. 
But note that some modalities of these logics are not first-order definable (by means of appropriate first-order languages describing Kripke structures) and 'contain' an expressive power that may be not seen if we consider propositional languages only. Therefore, to show some possibilities of the modalities we add them to the first-order classical language and then propose and discuss some facts concerning logics and classes of logics in resulting languages.

Mathematical results presented here, in fact, follow from constructions used to prove that some first-order logics defined by classes of Kripke frames are not recursively enumerable. So the reader may see on this paper as a discussion on just one of corollaries from such proofs.

\section{Decidability and recursive enumerability}

Here we just recall the notions of decidability and recursive enumerability. Let $U$ be some universal set (for our purposes it is sufficient $U$ to be the set of all formulas in a certain language) and let $X$ be a subset of $U$. Then $X$ is called decidable if there exists an algorithm $\mathfrak{A}$ such that, for any $x \in U$,

$$
\mathfrak{A}(x)= \begin{cases}1, & \text { if } x \in X \\ 0, & \text { if } x \notin X\end{cases}
$$

If $X$ is not a decidable set then it is called undecidable. The set $X$ is called recursive enumerable if $X=\varnothing$ or there exists an algorithm $\mathfrak{A}$ such that $X=\{\mathfrak{A}(n): n \in \mathbb{N}\}$, i. e., there exists an algorithm enumerating elements in $X$. Note also that $X$ is recursively enumerable if and only if there exists an algorithm $\mathfrak{A}$ such that, for any $x \in U$,

$$
\mathfrak{A}(x)= \begin{cases}\text { something, } & \text { if } x \in X, \\ \text { not defined, } & \text { if } x \notin X,\end{cases}
$$

i. e. $X$ is the domain of the algorithm $\mathfrak{A}$. For more details see $[8,13,20]$.

\section{Calculi}

Let us clarify what we mean by a calculus. Usually it is assumed that calculus is defined by a set of axioms and a set of inference rules. Both sets together, in fact, generate a set of derivable formulas. For our purposes it is important to be sure that such generation can be realized as an algorithmic procedure. Therefore we just add the following natural conditions: the set of axioms and the set of inference rules must be recursively enumerable and every inference rule must be realizable as an algorithm. The only 
property of calculi we are going to use is that the set of derivable formulas is recursively enumerable; it is ensured by the conditions.

Note, by the way, that any calculus with finite set of axioms and finite set of finitary inference rules, of course, satisfies both conditions above.

Below we sometimes equate a calculus to the set of all formulas derivable in it.

\section{Language under consideration}

Let us fix a language $\mathcal{L}$ containing a countable set of individual variables, a countable set of predicate letters of any arity (for every $m \in \mathbb{N}$, the language contains a countable set of $m$-ary predicate letters), $\wedge$ (conjunction), $\vee$ (disjunction), $\rightarrow$ (implication), $\neg$ (negation), quantifiers on individual variables $\forall x$ and $\exists x$ (for every variable $x$ ), modalities $\boldsymbol{A X}, \boldsymbol{A F}, \boldsymbol{E U}$, and technical symbols (comma and parentheses). In other words, we enrich the classical first-order language with the modalities of CTL. Formulas are constructed in the usual way: if $x_{1}, \ldots, x_{m}$ are variables, $P$ is $m$-ary predicate letter then $P\left(x_{1}, \ldots, x_{m}\right)$ is a formula; if $\varphi$ and $\psi$ are formulas and $x$ is a variable then $(\varphi \wedge \psi),(\varphi \vee \psi),(\varphi \rightarrow \psi), \neg \varphi, \forall x \varphi, \exists x \varphi, \boldsymbol{A X} \varphi, \boldsymbol{A F} \varphi$, and $(\varphi \boldsymbol{E} \boldsymbol{U} \psi)$ are formulas, too.

\section{Kripke semantics}

By a Kripke frame here we understand a triple $\mathfrak{F}=\langle W, R, D\rangle$ where $W$ is a non-empty set of states, $R$ is a serial binary accessibility relation on $W$, and $D$ is a function associating with every state $s$ its domain (i.e., some non-empty set of individuals) such that $D(s) \subseteq D(t)$ whenever $s R t$, for any $s, t \in W$. Kripke model on a frame $\mathfrak{F}$ is a pair $\mathfrak{M}=\langle\mathfrak{F}, I\rangle$ where $I$ is an interpretation of predicate letters in the domains of states, i.e., if $P$ is $n$-ary predicate letter and $s$ is a state then $I(s, P)$ is an $n$-ary relation on $D(s)$.

An infinite sequence $\pi=s_{0}, s_{1}, s_{2}, \ldots$ is called path in a frame $\mathfrak{F}=\langle W, R, D\rangle$ if, for any $k \in \mathbb{N}$, we have $s_{k} \in W$ and $s_{k} R s_{k+1}$. We assume that $\pi_{k}$ denotes the $k$-th element of the path $\pi$. We say that a path $\pi$ starts from a state $s$ if $\pi_{0}=s$. Note that, because $R$ is serial, for any $s \in W$, there is at least one path in $\mathfrak{F}$ starting from $s$.

Let $s$ be a state in a frame $\mathfrak{F}=\langle W, R, D\rangle$. A function $\alpha$ is called interpretation of individual variables in $s$ if $\alpha\left(x_{i}\right) \in D(w)$, for every individual variable $x_{i}$. 
Note that if $s^{\prime}$ is accessible from $s$ and $\alpha$ is an interpretation of individual variables in $s$ then $\alpha$ is an interpretation of individual variables in $s^{\prime}$, too, because in this case we have $D(w) \subseteq D\left(w^{\prime}\right)$.

For any individual variable $x$, let us define the binary relation $\stackrel{x}{=}$ on interpretations: for interpretations $\alpha$ and $\beta$ we put

$$
\alpha \stackrel{x}{=} \beta \leftrightharpoons \alpha(y)=\beta(y) \text {, for any variable } y \text { such that } y \neq x .
$$

Let $\mathfrak{M}=\langle\mathfrak{F}, I\rangle$ be a model on a serial frame $\mathfrak{F}=\langle W, R, D\rangle$. We define the truth relation 'a formula $\varphi$ is true at a state $s \in W$ in a model $\mathfrak{M}$ under an interpretation $\alpha$ of individual variables in $s^{\prime}$ inductively (by constructing of $\varphi$ ). We put

$$
(\mathfrak{M}, s) \models^{\alpha} P\left(x_{1}, \ldots, x_{m}\right) \leftrightharpoons\left\langle\alpha\left(x_{1}\right), \ldots, \alpha\left(x_{m}\right)\right\rangle \in I(s, P)
$$

where $P$ is $m$-ary predicate letter, $x_{1}, \ldots, x_{m}$ are individual variables. For other formulas the relation is defined as follows:

$$
\begin{aligned}
& (\mathfrak{M}, s) \models^{\alpha} \varphi_{1} \wedge \varphi_{2} \leftrightharpoons(\mathfrak{M}, s) \models^{\alpha} \varphi_{1} \text { and }(\mathfrak{M}, s) \models^{\alpha} \varphi_{2} ; \\
& (\mathfrak{M}, s) \models^{\alpha} \varphi_{1} \vee \varphi_{2} \leftrightharpoons(\mathfrak{M}, s) \models^{\alpha} \varphi_{1} \text { or }(\mathfrak{M}, s) \models^{\alpha} \varphi_{2} ; \\
& (\mathfrak{M}, s) \mid=^{\alpha} \varphi_{1} \rightarrow \varphi_{2} \leftrightharpoons(\mathfrak{M}, s) \not \nvdash^{\alpha} \varphi_{1} \text { or }(\mathfrak{M}, s) \models{ }^{\alpha} \varphi_{2} ; \\
& (\mathfrak{M}, s) \models^{\alpha} \neg \varphi_{1} \quad \leftrightharpoons(\mathfrak{M}, s) \not \models^{\alpha} \varphi_{1} ; \\
& (\mathfrak{M}, s) \models^{\alpha} \boldsymbol{A} \boldsymbol{X} \varphi_{1} \leftrightharpoons \text { for any path } \pi \text { starting from } s \text { the } \\
& \text { relation }\left(\mathfrak{M}, \pi_{1}\right) \models{ }^{\alpha} \varphi_{1} \text { is true; } \\
& (\mathfrak{M}, s) \models^{\alpha} \boldsymbol{A F} \varphi_{1} \leftrightharpoons \text { for any path } \pi \text { starting from } s \\
& \text { there is some } k \in \mathbb{N} \text { such that } \\
& \left(\mathfrak{M}, \pi_{k}\right) \mid=^{\alpha} \varphi_{1} \text {; } \\
& (\mathfrak{M}, s) \models{ }^{\alpha} \varphi_{1} \boldsymbol{E} \boldsymbol{U} \varphi_{2} \leftrightharpoons \text { for some path } \pi \text { starting in } s \\
& \text { and some } k \in \mathbb{N} \text { such that } \\
& \left(\mathfrak{M}, \pi_{k}\right) \models=^{\alpha} \varphi_{2} \text { and, for any } j \in \mathbb{N} \text {, } \\
& \text { such that } j<k \text { the relation } \\
& \left(\mathfrak{M}, \pi_{j}\right) \models{ }^{\alpha} \varphi_{1} \text { is true; } \\
& (\mathfrak{M}, s) \mid=^{\alpha} \forall x_{i} \varphi_{1} \leftrightharpoons \text { for any interpretation } \beta \text { such that } \\
& \beta \stackrel{x_{i}}{=} \alpha \text { and } \beta\left(x_{i}\right) \in D(s) \text { the relation } \\
& (\mathfrak{M}, s) \models^{\beta} \varphi_{1} \text { is true; }
\end{aligned}
$$




$$
\begin{aligned}
(\mathfrak{M}, s) \models^{\alpha} \exists x_{i} \varphi_{1} \leftrightharpoons & \text { there is an interpretation } \beta \text { such } \\
& \text { that } \beta \text { 丝 } \alpha, \beta\left(x_{i}\right) \in D(s), \text { and } \\
& (\mathfrak{M}, s) \models^{\beta} \varphi_{1} .
\end{aligned}
$$

As usual, a formula is said to be true in a model if it is true at any state in it; a formula is said to be true in a frame if it is true in any model on the frame; a formula is said to be true in a class of frames if it is true in any frame in the class.

Let us define the logic QCTL as the set of formulas that are true in the class of all (serial) Kripke frames.

\section{Kripke completeness}

We say that a set $L$ of formulas is Kripke complete if there is a class of Kripke frames such that $L$ coincides with the set of all formulas that are true in the class.

Note that if a set $L$ is Kripke complete then it is closed, at least, under modus ponens, generalization, and predicate substitution, i.e., $L$ may be viewed as a logic.

For example, QCTL is Kripke complete by its definition; any proper subset of QCTL is not Kripke complete (if we do not restrict the language and do not extend the class of frames, of course).

\section{Logic QCTLlinCD}

For some technical purposes we need to define a special extension of QCTL. We call a frame linear if the reflexive and transitive closure of its accessibility relation is linear. The frame $\langle W, R, D\rangle$ is said to be a frame with constant domains if $D(s)=D(t)$ whenever $s R t$, for any $s, t \in W$. Define QCTLlinCD as the set of formulas complete under the class of all linear frames with constant domains.

\section{Class of Kripke incomplete calculi}

Here we just propose and prove a statement which reflects the topic of the paper.

TheOREM 1. Let $S$ be a calculus such that $S \subseteq$ QCTLlinCD. Then $S$ is not Kripke complete.

Proof. Let us denote by $\mathbf{Q C L}_{f i n}$ the classical theory of finite models. In [15] it is proved that there is a translation $E m b$ such that, for any closed classical first-order formula $\varphi$,

$$
\varphi \in \mathbf{Q C L}_{f i n} \Longleftrightarrow \operatorname{Emb}(\varphi) \in \mathbf{Q C T L} .
$$


More exactly, it is shown that, for any closed classical first-order formula $\varphi$,

$$
\begin{aligned}
& \varphi \in \mathbf{Q C L}_{f i n} \Longrightarrow \operatorname{Emb}(\varphi) \in \mathbf{Q C T L} ; \\
& \varphi \notin \mathbf{Q} \mathbf{C L}_{f i n} \Longrightarrow \operatorname{Emb}(\varphi) \notin \mathbf{Q C T L l i n C D} .
\end{aligned}
$$

The second implication follows from the fact that if $\varphi \notin \mathbf{Q C L}_{\text {fin }}$ then $\operatorname{Emb}(\varphi)$ is refuted in a linear frame with constant domains (for details see $[15])$.

Because $\mathbf{Q C L}_{\text {fin }}$ is not recursively enumerable [6], from these two implications it immediately follows that any set of formulas between QCTL and QCTLlinCD is not recursively enumerable, too. Indeed, let $L$ be any set of formulas such that QCTL $\subseteq L \subseteq$ QCTLlinCD. Let $\varphi$ be a closed first-order formula. If $\varphi \in \mathbf{Q C L}_{\text {fin }}$ then, by the first implication $\operatorname{Emb}(\varphi) \in \mathbf{Q C T L}$, and hence, $\operatorname{Emb}(\varphi) \in L$; if $\varphi \notin \mathbf{Q C L}_{f i n}$ then, by the second implication, $\operatorname{Emb}(\varphi) \notin \mathbf{Q C T L l i n C D}$, and hence, $\operatorname{Emb}(\varphi) \notin L$. Therefore,

$$
\varphi \in \mathbf{Q C L}_{\text {fin }} \Longleftrightarrow \operatorname{Emb}(\varphi) \in L,
$$

and, as a corollary, $L$ is not recursively enumerable.

Suppose that $S$ is Kripke complete. Then QCTL $\subseteq S$. Together with the condition that $S \subseteq$ QCTLlinCD it means that QCTL $\subseteq S \subseteq$ QCTLlinCD, and hence it is not recursively enumerable. But this is impossible because $S$ is a calculus. From the contradiction it follows that $S$ is not Kripke complete. The theorem is proved.

\section{Discussion}

Now we have got a matter for our discussion: the theorem and its proof. Both the theorem and the proof are quite short but we want to show some hidden details.

\subsection{Examples of Kripke incomplete calculi}

First of all, we give an explanation how to apply the theorem. Suppose we have some calculus $S$ with a set of axioms $\mathcal{A}$ and a set of inference rules $\mathcal{R}$. Suppose also that we are able to check, for every $\varphi \in \mathcal{A}$, whether $\varphi$ is true in the class of all linear frames with constant domains and, for every rule in $\mathcal{R}$, whether the rule is admissible in the same class (note, by the way, that there is no general procedure solving these tasks [15] but sometimes it is not the case for particular calculi). Then, if for every axiom and every rule the check is OK, then by the theorem we may conclude that $S$ is not Kripke complete. 
We give an example. Let $\mathcal{A}=\mathbf{C T L} \cup \mathbf{Q C L}$, where $\mathbf{Q C L}$ is the classical first-order logic, and let $\mathcal{R}$ be consisting of modus ponens, generalization, and substitution. Then the calculus defined by $\mathcal{A}$ and $\mathcal{R}$ is not Kripke complete.

If we extend the calculus with any formulas that are true in linear frames with constant domains (for example, bounded width formulas, bounded branching formulas, Barcan formula, etc.) and inference rules preserving validity in all such frames (for example, necessitation rule for the modality $\boldsymbol{A} \boldsymbol{X}$ ) then we again obtain a Kripke incomplete calculus.

\subsection{Possibility of constructive proofs for the theorem}

Note that the proof presented here is not constructive: to prove the theorem we suppose it to be wrong and then obtain a contradiction. To give a constructive proof we must construct a formula $\varphi$ that is not derivable in $S$ but true in any Kripke frame for $S$.

Obviously, there is no such a formula for all calculi. Indeed, suppose $\varphi$ is not derivable in any calculus $S$ such that $S \subseteq$ QCTLlinCD but $\varphi \in$ QCTLlinCD. If we add $\varphi$ to $S$ as an extra axiom then we obtain a calculus included into QCTLlinCD and containing $\varphi$, that gives us a contradiction.

Therefore, to get a constructive proof we need an effective procedure finding, for any calculus $S$ such that $S \subseteq$ QCTLlinCD, a formula $\varphi_{S}$ such that $\varphi_{S} \in$ QCTLlinCD but $\varphi_{S}$ is not derivable in $S$. The problem is in that the set of all such calculi (i. e., in fact, the set of inputs for the procedure) is not effectively definable because it is not recursively enumerable. Indeed, let $S_{\varphi}=\{\varphi\}$, for every formula $\varphi$ (i. e., $S_{\varphi}$ is a calculus with one axiom and without inference rules). Then the set $\left\{S_{\varphi}: S_{\varphi} \subseteq\right.$ QCTLlinCD $\}$ coincides with the set $\{\{\varphi\}: \varphi \in$ QCTLlinCD $\}$ and hence it is not recursively enumerable. The same argumentation (with slight modifications) works also for calculus containing QCL and closed under some 'natural' inference rules; we leave details to the reader.

Nevertheless, of course, there are constructive ways to prove nearly the same theorem. Because any formula is constructed effectively, by the theorem we have that for any calculus $S$ such that $S \subseteq$ QCTLlinCD there is an algorithm constructing a formula $\varphi_{S}$ such that $\varphi_{S} \in$ QCTLlinCD but $\varphi_{S}$ is not derivable in $S$. Therefore, for any particular calculus $S$ there is a constructive proof of its Kripke incompleteness. Clearly, if $S^{\prime} \subseteq S$ and $\varphi$ is not derivable in $S$ then $\varphi$ is not derivable in $S^{\prime}$, too. Hence, we may replace QCTLlinCD in the theorem with any particular calculus and then obtain a constructive proof. For example, instead of QCTLlinCD we may 
take a calculus containing QCL, CTL, linearity axiom, Barcan formula and closed under substitution, modus ponens, generalization, and maybe some other inference rules.

We did not try to obtain a constructive proof this way, and here we leave the details of the question to the reader.

\subsection{Extensions of the language}

Note that CTL is a fragment of $\mathbf{C T L}{ }^{*}$ and, modulo some translation, it is also a fragment of ATL and $\mathbf{A T L}{ }^{*}$. It means that we can repeat our argumentation for logics QCTL*, QATL, QATL* (the reader may define them using corresponding Kripke semantics for $\mathbf{C T L}{ }^{*}$, ATL, ATL*). But, in fact, we do not need it: it is enough to use the theorem for calculi in extended language. Let us understand $\mathcal{L}$-fragment of a calculus (in a language extending $\mathcal{L}$ ) as a set consisting of all formulas derivable in the calculus that are in $\mathcal{L}$ (maybe, modulo a certain translation).

Corollary 1. Let $S$ be a calculus in the language of QCTL*, QATL or QATL* $^{*}$ such that the $\mathcal{L}$-fragment of $S$ is a subset of QCTLlinCD. Then $S$ is not Kripke complete.

Moreover, we may imagine a situation when we deal with some different language allowing to express $\mathcal{L}$ inside of it. Then the corollary is true, too.

\subsection{Fragments of the language}

Now let us turn to another 'direction', and put the following question: what happens if we restrict $\mathcal{L}$ ?

Due to S. Kripke [16], if we restrict $\mathcal{L}$ with just unary predicate letters then both the theorem and the corollary are still true; moreover, we propose a hypothesis that sometimes even one unary letter is enough [3, 4]. As for individual variables, we think that three ones are enough; maybe even two [14]. But here we discuss the modalities, therefore we consider some restrictions on their using.

In accordance with literature on CTL, we distinguish five 'basic' modalities: $\boldsymbol{A X}, \boldsymbol{A} \boldsymbol{G}, \boldsymbol{A F}, \boldsymbol{E U}$, and $\boldsymbol{A U}$. Formally, the language $\mathcal{L}$ already contains $\boldsymbol{A} \boldsymbol{X}, \boldsymbol{A F}$, and $\boldsymbol{E} \boldsymbol{U}$, therefore we define just $\boldsymbol{A} \boldsymbol{G}$ and $\boldsymbol{A} \boldsymbol{U}$ : $\boldsymbol{A} \boldsymbol{G} \varphi=\neg \boldsymbol{E}((\varphi \rightarrow \varphi) \boldsymbol{U} \neg \varphi), \boldsymbol{A}(\varphi \boldsymbol{U} \psi)=\boldsymbol{A} \boldsymbol{F} \varphi \wedge \neg \boldsymbol{E}(\neg \psi \boldsymbol{U}(\neg \varphi \wedge \neg \psi))$. Note that we also may define five dual modalities (known as $\boldsymbol{E} \boldsymbol{X}, \boldsymbol{E} \boldsymbol{F}$, $\boldsymbol{E} \boldsymbol{G}, \boldsymbol{A R}$, and $\boldsymbol{E} \boldsymbol{R})$ but they are not essential for our purposes, and we leave details to the reader $[1,9,17]$. Every subset of the set $\{\boldsymbol{A X}, \boldsymbol{A G}, \boldsymbol{A F}, \boldsymbol{E} \boldsymbol{U}, \boldsymbol{A U}\}$ defines a certain fragment of QCTL, and we consider such fragments. 
Let $M$ be a subset of $\{\boldsymbol{A} \boldsymbol{X}, \boldsymbol{A} \boldsymbol{G}, \boldsymbol{A F}, \boldsymbol{E} \boldsymbol{U}, \boldsymbol{A} \boldsymbol{U}\}$. For a set $L$ of formulas, define $L \uparrow M$ as a fragment of $L$ where only modalities contained in $M$ are used. For example, QCTL $\lceil\varnothing=\mathbf{Q C L}$. In fact, in [15] it is proved that from QCTL $\subseteq L \subseteq$ QCTLlinCD, $M \neq \varnothing, M \neq\{\boldsymbol{A} \boldsymbol{X}\}$, and $M \neq\{\boldsymbol{A} \boldsymbol{G}\}$ it follows that $L \uparrow M$ is not recursively enumerable. Hence, for such fragments we may use the same argumentation as in the proof of the theorem. As a result we obtain the following proposition.

Proposition 1. Let $M$ be a set of modalities allowing to express at least one of the modalities $\boldsymbol{A F}, \boldsymbol{E U}, \boldsymbol{A U}$ or both $\boldsymbol{A X}$ and $\boldsymbol{A} \boldsymbol{G}$, let also $S$ be a calculus such that $S \uparrow M \subseteq \mathbf{Q C T L l i n C D} \uparrow M$; then $S$ is not Kripke complete.

This proposition is stronger than the theorem. It shows that just one temporal modality may be quite expressive. But note that the proposition does not tell us anything about calculi in the language with $\boldsymbol{A} \boldsymbol{X}$ only and with $\boldsymbol{A} \boldsymbol{G}$ only.

\subsection{Effect of first-order conditions}

To define Kripke semantics for $\mathcal{L}$ we need the notion of path. Formally, $\pi$ is a path in a frame $\langle W, R, D\rangle$ if $\pi$ is a map from $\mathbb{N}$ to $W$ such that $\pi(n) R \pi(n+1)$, for every $n \in \mathbb{N}$. Then, to define the truth relation for the modalities, in fact, we use second-order quantifiers (on paths).

It is not the case for $\boldsymbol{A} \boldsymbol{X}$. It is possible to define the truth relation for it using just $R$ and first-order quantifiers (on states in $W$ ): $\boldsymbol{A} \boldsymbol{X} \varphi$ is true at a state $s$ if $\varphi$ is true at every state $t$ such that $s R t$. Together with first-order definability of seriality and linearity it provides us with embeddings of QCTL $\uparrow\{\boldsymbol{A X}\}$ and QCTLlinCD $\uparrow\{\boldsymbol{A X}\}$ into QCL, and hence with a recursive axiomatization for each of them. Of course, it is not so for any logic between QCTL $\uparrow\{\boldsymbol{A X}\}$ and QCTLlinCD $\uparrow\{\boldsymbol{A X}\}$ into QCL but from the construction in [5] we obtain the following observation: let a logic $L$ be Kripke complete under some first-order definable class of frames; then $L\lceil\{\boldsymbol{A X}\}$ is recursively enumerable. Note that any recursively enumerable logic has also a recursive axiomatization [10]. Note also that we do not know whether the converse statement for the observation holds.

To define the truth relation for $\boldsymbol{A G}$, in fact, we must define reflexive and transitive closure of arbitrary accessibility relation $R$. It is not possible to define it via $R$ and equality if we use the first-order language only, but note that if we deal with the modality $\boldsymbol{A} \boldsymbol{G}$ without all others, then we may 'forget' about $R$ and use its reflexive and transitive closure as a unique binary relation in a frame. In this case, we must just claim it to be reflexive 
and transitive. Then, we are again in the similar situation: there are embeddings of QCTL $\uparrow\{\boldsymbol{A G}\}$ and QCTLlinCD $\uparrow\{\boldsymbol{A G}\}$ into QCL, wherefore these fragments (and some ones between them) are recursively axiomatizable.

Of course, if we consider classes of frames allowing us to define other modalities using first-order conditions only, then we obtain recursively axiomatizable extensions of QCTL. For example, if a logic $L$ is complete under a class consisting of all frames $\langle W, R, D\rangle$ with the same finite $W$ and the same $R$ on it then $L$ is recursively axiomatizable.

\subsection{Other classes}

Our main conclusions and observations are based on the fact that any set between QCTL and QCTLlinCD is not recursively enumerable. What about logics outside the interval? In general, we do not know. But we show some difficulties. To do this, consider an example.

Let QCTLfin be the logic complete under the class of all finite Kripke frames. This logic is not included into QCTLlinCD but using argumentation as in [22] we obtain that QCTLfin is not recursively enumerable (and even QCTLfin $\uparrow\{\boldsymbol{A X}\}$ is not recursively enumerable). To prove the theorem as above but with QCTLfin instead of QCTLlinCD we need an algorithm embedding some non-enumerable problem into both QCTL and QCTLfin, simultaneously. But we do not know whether such an algorithm exists.

Let QCTLfinCD be the logic complete under the class of all finite Kripke frames with constant domains. It is not recursively enumerable, too. Moreover, it is possible to show that there is a translation $t r$ such that, for any closed classical first-order formula $\varphi$,

$$
\begin{aligned}
\varphi \in \text { QCTLfin } & \Longleftrightarrow \operatorname{tr}(\varphi) \in \text { QCTLfin } \\
& \Longleftrightarrow \operatorname{tr}(\varphi) \in \text { QCTLfinCD },
\end{aligned}
$$

and hence, any set of formulas between QCTLfin and QCTLfinCD is not recursively enumerable (unfortunately, we do not know about any publications containing this fact, and cannot give a reference). It seems we are successful and can extend the class of Kripke incomplete calculi. But this is not so, again. Indeed, in this case, we just may propose that any calculus such that QCTLfin $\subseteq S \subseteq$ QCTLfinCD is not Kripke complete but, clearly, there is no such calculus. 


\subsection{Impossibility of 'converse' embeddings}

In fact, our proof of the theorem is based on the fact that there exists an embedding of $\mathbf{Q C L}_{\text {fin }}$ into any theory between QCTL and QCTLlinCD. The following natural question arises: is there an effective embedding of QCTL or QCTLlinCD into $\mathbf{Q C L}_{\text {fin }}$ ? The answer is 'no', and here we give some argumentation.

Let us recall Post theorem, see [2]:

- a set $X$ is decidable if and only if both $X$ and $\bar{X}$ are recursively enumerable,

where $\bar{X}$ is the complement of $X$. Clearly, for a logic $L$ and a formula $\varphi$, we have that

- $\varphi$ is $L$-valid if and only if $\neg \varphi$ is not $L$-satisfiable;

- $\varphi$ is not $L$-satisfiable if and only if $\neg \varphi$ is $L$-valid.

Therefore, in terms of $L$-validity and $L$-satisfiability, Post theorem means that

- $L$ is decidable if and only if both L-validity problem and L-satisfiability problem are recursively enumerable,

and hence, if $L$ is undecidable then at least one of the problems is not recursively enumerable.

The following statement is known as Church theorem, see [8, 13]: QCL is not decidable. But because QCL is finitely axiomatizable, it is also recursively enumerable. Therefore, using Post theorem, we may conclude that

- QCL-validity problem is recursively enumerable;

- QCL-satisfiability problem is not recursively enumerable.

Note also that the set of all $\mathbf{Q C L} \mathbf{L}_{f i n}$-satisfiable formulas is recursively enumerable: corresponding algorithm just tests for a given formula whether it is true in models with one element, then whether it is true in models with two elements, then whether it is true in models with three elements, and so on; if the algorithm finds a model satisfying the formula then it stops with the positive answer. Therefore, we may specify Trakhtenbrot theorem [6]:

- $\mathbf{Q C L}_{f i n}$-validity problem is not recursively enumerable; 
- $\mathbf{Q C L}_{f i n}$-satisfiability problem is recursively enumerable.

Observe that for any closed classical first-order formula $\varphi$,

$$
\begin{aligned}
\varphi \in \text { QCL } & \Longleftrightarrow \varphi \in \text { QCTL } \\
& \Longleftrightarrow \varphi \in \text { QCTLlinCD. }
\end{aligned}
$$

Because QCL-satisfiability problem is not recursively enumerable, as a corollary we obtain that $L$-satisfiability problem is not recursively enumerable, too, for any logic between QCTL and QCTLlinCD (of course, the same is also true for any logic between QATL and QATLlinCD, etc.).

Let $L$ be a logic between QCTL and QCTLlinCD. Suppose that there exists an embedding of $L$ into $\mathbf{Q} \mathbf{C L}_{\text {fin }}$, i.e., there exists an algorithm $A$ such that

$$
\varphi \in L \Longleftrightarrow A(\varphi) \in \mathbf{Q C L}_{\text {fin }},
$$

for any formula $\varphi$ in the language $\mathcal{L}$. Then we immediately obtain that $\mathbf{Q C L}_{f i n}$-satisfiability problem is not recursively enumerable but it is not so. The contradiction means that there is no such embedding. The same argumentation allows us to conclude that there is no embedding of $L$ into QCL, too.

\section{Acknowledgements}

Our thanks to participants of the conference LARA-2014, where these results were presented and discussed, for their attention and questions. We are also grateful to anonymous reviewer for remarks and advises; in fact, section 9.2 was added because of one of the remarks.

\section{References}

[1] Karpov, Yu.G. Model checking: Verifikatsiya parallel'nykh $i$ raspredelennykh programmnykh sistem [Model checking: Verification of parallel and distributed software systems]. Sankt-Peterburg: BKhV-Peterburg, 2010. 560 pp. (In Russian)

[2] Mal'tsev, A.I. Algoritmy $i$ rekursivnye funktsii [Algorithms and recursive functions]. M.: Nauka, Fizmatlit, 1986. 367 pp. (In Russian)

[3] Maslov, Yu. S., Mints E. S., Orevkov V. P. 'Nerazreshimost' v konstruktivnom ischislenie predikatov nekotorykh klassov i formul, soderzhashchikh tol'ko odnomestnye predikatnye peremennye" [Unsolvability in constructive predicate calculus certain classes of formulas containing only single predicate variables], Doklady AN SSSR [Reports of the Academy of Sciences USSR], 1965, vol. 163, no 2, pp. 295-297. (In Russian) 
[4] Rybakov, M.N. "Ob algoritmicheskoi vyrazitel'nosti modal'nogo yazyka s odnoi lish' odnomestnoi predikatnoi bukvoi" [About algorithmic expressiveness modal language with only one single predicate letter], Logicheskie issledovaniya [Logical investigations]. M.: Nauka, 2002, vol.9, pp. 179-201. (In Russian)

[5] Rybakov, M.N., Chagrov, A.V. "Standartnye perevody neklassicheskikh formul i otnositel'naya razreshimost' logik" [Standard formulas and transfers of nonclassical logics relative solvability], Trudy nauchno-issledovatel'skogo seminara Logicheskogo tsentra Instituta Filosofii RAN [Proceedings of the Research Seminar Logic Center Institute of Philosophy RAS]. M.: Izdatel'stvo Instituta Filosofii RAN, 2000, vol. XIV, pp. 81-98. (In Russian)

[6] Trakhtenbrot, B.A. "Nevozmozhnost' algoritma dlya problemy razreshimosti na konechnykh klassakh" [The impossibility of an algorithm for the solvability of the problem in the final classes], Doklady Akademii Nauk SSSR [Reports of the Academy of Sciences USSR], 1950, vol. 70, no 4, pp. 596-572. (In Russian)

[7] Alur, R., Henzinger, T.A., Kupferman, O. "Alternating-time temporal logic", Journal of the ACM, 2002, vol. 19, no 5, pp. 672-713.

[8] Boolos, G.S., Burgess, J.P., Jeffrey, R.C. Computability and Logic. Cambridge: Cambridge University Press, 2007. 366 pp.

[9] Clarke, E.M., Grumberg, O., Peled, D.A. Model Checking. Cambridge: The MIT Press, 1999. 314 pp.

[10] Craig, W. "On axiomatizability within a system", The Journal of Symbolic Logic, 1953, vol. 18, no 1, pp. 30-32.

[11] Emerson, E.A., Halpern, J.Y. "Decision procedures and expressiveness in the temporal logic of branching time", Journal of Computer and System Sciences, 1985, no 30(1), pp. 1-24.

[12] Emerson, E.A., Julta, C.S. "The complexity of tree automata and logics of programs", In Proceedings of the 29th Annual Symposium on Foundations of Computer Science, 1988, pp. 328-337.

[13] Kleene, S.C. Mathematical Logic. John Wiley \& Sons, Inc: New York, London, Sydney, 1967. $432 \mathrm{pp}$.

[14] Konchakov, R., Kurucz, A., Zakharyaschev, M. "Undecidability of first-order intuitionistic and modal logics with two variables", Bulletin of Symbolic Logic, 2011, vol. 11, pp. 428-438.

[15] Kotikova, E.A., Rybakov M.N. "First-Order Logics of Branching Time: On Expressive Power of Temporal Operators", Logical Investigations, 2013, vol. 19, pp. 68-99.

[16] Kripke, S. "The Undecidability of Monadic Modal Quantificational Theory", Zeitschrift für Mathematische Logik und Grundlagen der Mathematik, 1962, vol. 8, pp. 113-116.

[17] Laroussinie, F. "About the expressive power of CTL combinators", Information Processing Letters, 1995, no 54(6), pp. 343-345. 
[18] Prior, A.N. Past, present and future. Oxford: Oxford University press, 1967. 228 pp.

[19] Schewe, S. "ATL* satisfiability is 2EXPTIME-complete", in: Automata, Languages, and Programming, ed. by L. Aceto et al. 2008, vol. 5126 of Lecture Notes in Computer Science, pp. 373-385.

[20] Shoenfield, J.R. Degrees of Unsolvability. North-Holland Publishing Company: American Elsevier Publishing Company, 1971.

[21] Sistla, A.P., Clarke, E.M. "The Complexity of Propositional Linear Temporal Logics", Journal of the Association for Computing Machinery, 1985 (July), vol. 32, no 3, pp. 733-749.

[22] Skvortsov, D. "On The Predicate Logics of Finite Kripke Frames", Studia Logica, 1995, vol. 54, pp. 79-88.

[23] Vardi, M.Y., Stockmeyer, L. "Improved upper and lower bounds for modal logics of programs: Preliminary report", In Proceedings of the seventeenth annual ACM symposium on Theory of computing, 1985, pp. 240-252.

[24] Walther D., Lutz C., Walter F., Wooldridge M. "ATL satisfiability is indeed EXPTIME-complete", Journal of Logic and Computation, 2006, no 16(6), pp. 765-787. 